\title{
Analysis of Optimal Diesel-electric Powertrain Transients during a Tip-in Maneuver
}

\author{
Vaheed Nezhadali Lars Eriksson \\ Vehicular Systems Division, Electrical Engineering Department \\ Linköping University, SE-581 83 Linköping, Sweden \{vaheed.nezhadali, lars.eriksson\}@liu.se
}

\begin{abstract}
Optimal transients of a hybrid powertrain are calculated with the aim to give a smooth and time efficient acceleration. It is shown that there is a trade-off between time and driveline oscillations where high oscillations can be avoided by slightly longer acceleration time and proper control of the electrical and diesel power sources. During a low oscillation acceleration, there is still the possibility to reduce the amount of total consumed electrical and fuel energy. This is investigated by calculation of optimal controls during acceleration for a fixed time while penalizing the usage of energy in a low oscillation acceleration. The balance between electrical and diesel energy usage during the acceleration is also investigated. The results show that to avoid extreme transients by optimal control, a multidimensional formulation of the objective function including different properties should be considered.
\end{abstract}

Keywords: numerical optimal control, acceleration, vehicle jerk

\section{Nomenclature}

The nomenclature for the paper with subscripts and variables is given in Tables 1 and 2 respectively.

Table 1. Subscripts used for variables.

\begin{tabular}{cc|cc}
\hline Index & Description & Index & Description \\
\hline im & Intake manifold & $e m$ & Exhaust manifold \\
gen & Generator & $w g$ & Wastegate \\
$e$ & Engine & $a$ & Air \\
$d s$ & Drive shaft & $v$ & Vehicle \\
$m f$, conv & Fuel conversion & $m_{f}$ & Fuel mass \\
loss & Losses & $t o t$ & Total \\
$m e c h$ & Mechanical & $t c$ & Turbocharger \\
$w$ & Wheel & $g b$ & Gearbox \\
$f d$ & Final drive & $r e s i s t$ & Resistant forces \\
$c$ & Compressor & $a c$ & Air into cylinder \\
0 & Initial & $f$ & Final \\
$r$ & Radius & $g s$ & Genset \\
\hline
\end{tabular}

\section{Introduction}

Hybridization of powertrains opens up new opportunities for faster and more efficient vehicle acceleration. With an electric power source assisting a diesel engine, there is an extra degree of freedom in powertrain control while
Table 2. Variables used in the paper.

\begin{tabular}{|c|c|c|}
\hline Symbol & Description & Unit \\
\hline$x$ & State variable & - \\
\hline$u$ & Control input & - \\
\hline$\theta$ & Angle & $\mathrm{rad}$ \\
\hline$t$ & Time & $\mathrm{s}$ \\
\hline$F$ & Force & $\mathrm{N}$ \\
\hline$R$ & Gas constant & $\mathrm{N} \cdot \mathrm{m} / \mathrm{kg} \cdot \mathrm{K}$ \\
\hline$p$ & Pressure & $\mathrm{Pa}$ \\
\hline$T$ & Temperature & K \\
\hline$M$ & Torque & $\mathrm{N} \cdot \mathrm{m}$ \\
\hline$k$ & Stiffness coefficient & $\mathrm{N} \cdot \mathrm{m} / \mathrm{rad}$ \\
\hline$b$ & Damping coefficient & $\mathrm{N} \cdot \mathrm{m} \cdot \mathrm{s} / \mathrm{rad}$ \\
\hline$\omega$ & Rotational speed & $\mathrm{rad} \cdot \mathrm{s}^{-2}$ \\
\hline$\alpha$ & Rotational acceleration & $\mathrm{rad} / \mathrm{s}$ \\
\hline$\beta$ & Road slope & $\mathrm{rad}$ \\
\hline$m, \dot{m}$ & Mass, Mass flow & $\mathrm{kg}, \mathrm{kg} / \mathrm{s}$ \\
\hline$P$ & Power & W \\
\hline$E$ & Energy & $\mathrm{J}$ \\
\hline$u_{m f}, u_{w g}, P_{g e n}$ & Control signals & mg/cycle, -, W \\
\hline$J$ & Inertia & $\mathrm{kg} \cdot \mathrm{m}^{2}$ \\
\hline$\rho$ & Density & $\mathrm{kg} \cdot \mathrm{m}^{-3}$ \\
\hline$r$ & Radius & $\mathrm{m}$ \\
\hline$A$ & Vehicle frontal area & $\mathrm{m}^{2}$ \\
\hline$B S R$ & Blade speed ratio & - \\
\hline$\lambda$ & Air/fuel equivalence ratio & - \\
\hline$\phi$ & Fuel/air equivalence ratio & - \\
\hline$i$ & Gear ratio & - \\
\hline$\eta$ & Efficiency & - \\
\hline$\Pi$ & Compression ratio & - \\
\hline$c$ & Constant coefficient & - \\
\hline$\psi$ & Electrical energy penalty coefficient & - \\
\hline$\delta$ & Energy penalty coefficient & - \\
\hline$(A / F)_{s}$ & Stoichiometric Air to fuel ratio & - \\
\hline
\end{tabular}

the simultaneous control of the diesel and electric power sources becomes more complex.

Tip-in maneuver is referred to the situation where the driver suddenly asks for a fast vehicle acceleration by pressing accelerator pedal. This is a highly demanding and transient operation in a diesel-electric powertrain. The controls during this period can be optimized with respect to energy consumption or the operations time similar to (Sivertsson and Eriksson, 2012b), (Sivertsson and Eriksson, 2012a) and (Sivertsson and Eriksson, 2015b). Passenger comfort is also important when considering powertrain transients and can be accounted for by taking the driveline oscillations, referred to as Jerk, into account. The Jerk is also important considering its effects on the life length of driveline components, for more discussion see (Haj-Fraj and Pfeiffer, 2001) and (Haj-Fraj and Pfeiffer, 2002). In real world applications, not a single but all of these ob- 
jectives are of importance and therefore it is desirable to obtain a compromise between these objectives by proper control of the powertrain.

The contribution of this paper is the development of a methodology for the calculation of efficient hybrid powertrain transients with the aim to obtain a compromise between time-energy-Jerk objectives during a tip-in maneuver. Numerical optimal control is used as an enabler for this where first the extreme transients obtained by improper objective function formulations are presented. Then the trade-off between time-Jerk and Jerk-energy are calculated. The problem is solved for different road slopes representing various loading scenarios. The analysis is extended by investigation of powertrain transients and the balance between usage of diesel and electric energy sources is analyzed.

\section{Powertrain model}

To enable optimal control problem (OCP) formulation, a model for the powertrain and driveline components is needed. The powertrain model representing a hybrid bus is comprised of a diesel engine and an electric motor/generator working in parallel. The dynamics are described by a mean value engine model (MVEM) and a model for generator efficiency in a validated dieselelectric powertrain (genset) model from (Sivertsson and Eriksson, 2014). The powertrain dynamics are described by four state variables as $\omega_{e}(t), p_{i m}(t), p_{e m}(t)$ and $\omega_{t c}(t)$. Two additional states describe the dynamics of the driveshaft twist angle $\theta_{d s}(t)$ and wheel speed $\omega_{w}(t)$. The model has three control inputs for injected fuel during each combustion cycle $u_{m f}(t)$, wastegate position $u_{w g}(t)$ and the the electric power of motor/generator $P_{g e n}(t)$.

Dynamics of the four genset state variables are described by the following differential equations:

$$
\begin{aligned}
\frac{d \omega_{e}}{d t} & =\frac{1}{J_{g s}}\left(M_{g s}-M_{g s, l o a d}\right) \\
\frac{d p_{i m}}{d t} & =\frac{R_{i m} T_{i m}}{V_{i m}}\left(\dot{m}_{c}-\dot{m}_{a c}\right) \\
\frac{d p_{e m}}{d t} & =\frac{R_{e m} T_{e m}}{V_{e m}}\left(\dot{m}_{a c}+\dot{m}_{f}-\dot{m}_{t}-\dot{m}_{w g}\right) \\
\frac{d \omega_{t c}}{d t} & =\frac{P_{t} \eta_{m e c h}-P_{c}}{\omega_{t c} J_{t c}}
\end{aligned}
$$

The flexibilities in the driveline are lumped into one single flexibility in the driveshaft according to (Pettersson and Nielsen, 2000), and the torque transferred by the driveshaft is described using the stiffness and damping coefficients as follows:

$$
\begin{aligned}
M_{d s} & =k_{d s} \theta_{d s}+b_{d s} \frac{d \theta_{d s}}{d t} \\
\frac{d \theta_{d s}}{d t} & =\frac{\omega_{e}}{i_{g b} i_{f d}}-\omega_{w}
\end{aligned}
$$

where (6) is used to describe the driveshaft deflection dynamics.
Considering rolling and aerodynamic resistances and gravitational force, as well as constant gearbox and final drive ratios, the wheel speed dynamics are calculated using Newton's second law of motion as follows:

$$
\begin{aligned}
\frac{d \omega_{w}}{d t} & =\frac{M_{d s}-M_{\text {resist }}}{J_{w}+m_{v} r_{w}^{2}} \\
M_{\text {resist }} & =0.5 \rho_{\text {air }} c_{a} A \omega_{w}^{2} r_{w}^{3}+m_{v} g r_{w}\left(c_{r} \cos (\alpha)+\sin (\alpha)\right)
\end{aligned}
$$

The utilized electric and diesel energy are represented by the following integral states:

$$
\begin{aligned}
E_{\text {gen }} & =\int_{t_{0}}^{t_{f}} P_{g e n} d t \\
E_{m_{f}} & =q_{h v} \int_{t_{0}}^{t_{f}} u_{m f} \omega_{e} n_{c y l} \frac{10^{-6}}{4 \pi} d t
\end{aligned}
$$

When formulating OCPs, the oscillations in the rotational speed of the transmission shaft is used to represent the driveline oscillation. These oscillations are referred to as Jerk that is defined as follows:

$$
\begin{aligned}
\text { Jerk } & =\int_{t_{0}}^{t_{f}} \dot{\alpha}_{t r}^{2} d t \\
\alpha_{t r} & =\frac{d \omega_{e}}{d t} \frac{1}{i_{g b}}
\end{aligned}
$$

\section{Problem formulation}

In this section, first definition of the tip-in problem in terms of boundary conditions and constraints is described and then the objective function formulation for the OCPs are presented.

\subsection{Tip-in problem constraints}

\subsubsection{Boundary conditions for the tip-in problem}

The tip-in starts from a stationary operating condition at constant vehicle speed of $10 \mathrm{~km} / \mathrm{h}$ and the final condition is that the speed should reach $15 \mathrm{~km} / \mathrm{h}$. The states and control inputs should remain within the allowed limits during the operation while the integral states and generator power are assumed to be zero at the beginning. All these can be summarized as:

$$
\left\{\begin{array}{l}
\omega_{w}\left(t_{0}\right)=\frac{\omega_{e}\left(t_{0}\right)}{i_{f d \times g b}}=\frac{10}{r_{w}} \frac{1}{3.6}, \quad \dot{x}\left(t_{0}\right)=0, \\
\omega_{w}\left(t_{f}\right)=\frac{15}{r_{w}} \frac{1}{3.6}, \\
E_{g e n}\left(t_{0}\right)=E_{m_{f}}\left(t_{0}\right)=P_{\text {gen }}\left(t_{0}\right)=0, \\
u_{\min } \leq u \leq u_{\max }, x_{\min } \leq x \leq x_{\max }
\end{array}\right.
$$

\subsubsection{Path constraints during tip-in}

The problem is solved for a hybrid bus where the maximum acceleration of $1 \mathrm{~m} / \mathrm{s}^{2}$ according to the limits in SORT (Standardised On-Road Test cycles (SORT), Last 
accessed April 2016) are used as the highest allowed acceleration. The SORT standard is used in Europe to design on-road test cycles in order to measure fuel consumption of buses. There are also constraints regarding the turbocharger operation to avoid surge, and operational region for the turbine blade speed ratio. The maximum engine power is limited according to the maximum power curve at different engine speeds and finally, the air to fuel ratio should satisfy the smoke limit constraint $\lambda_{\min }$. There is also a mechanical limit on how fast the wastegate can be actuated and the rate of change in generator power. These constraints are summarized as:

$$
\left\{\begin{array}{l}
\frac{d \omega_{w}}{d t} \times r_{w}<1, \Pi_{c} \leq \Pi_{c, s u r g e}, \\
B S R_{\min } \leq B S R(x, u) \leq B S R_{\max }, \\
P_{e}(x, u) \leq P_{e, \max }(x), \frac{\dot{m}_{a c}}{\dot{m}_{f}}(A / F)_{s} \leq \frac{1}{\lambda_{\min }}, \\
\left|\dot{u}_{w g}\right| \leq c_{w g},\left|\dot{P}_{g e n}\right| / \omega_{e} \leq c_{g e n}
\end{array}\right.
$$

\subsection{Optimal control problem formulation}

In analysis of powertrain dynamics during tip-in, one objective is to calculate the minimum time transients of the powertrain. For this, OCPs with objective function of the following form are solved:

$$
\min _{(x, u)} \int_{t_{0}}^{t_{f}} d t
$$

The trade-off between minimum time and minimum Jerk transients will be calculated by first calculating the shortest time via solving the minimum time problem, and then, minimizing the Jerk using a fixed $t_{f}$. The time is then increased step wise compared to the calculated minimum time duration. The OCP formulation in this case looks as follows:

$$
\min _{(x, u)} \int_{t_{0}}^{t_{f, f i x}} \operatorname{Jerk} d t
$$

The energy from fuel and electrical sources during the vehicle acceleration can be minimized solving for:

$$
\min _{(x, u)} \int_{t_{0}}^{t_{f, f i x}}\left(E_{m_{f}}+E_{g e n}\right) d t
$$

In (Nezhadali and Eriksson, 2016) it is discussed that after minimizing the Jerk in a fixed time OCP, energy consumption minimization is the next dimension that can be analyzed for a low Jerk solution. This is done by calculating the Jerk optimal control transients including a penalty $\delta$ on energy consumption while using a fixed time. The penalty on the energy consumption is increased iteratively and the problem is solved several times to obtain the tradeoff between Jerk and energy objectives. The objective function formulation for this case is:

$$
\min _{(x, u)} \int_{t_{0}}^{t_{f, f i x}} \operatorname{Jerk}+\delta \times\left(E_{m_{f}}+E_{\text {gen }}\right) d t
$$

There is still another dimension to the optimization problem which is the balance between usage of diesel and electrical energy during the acceleration. To investigate how such balance affects the system transients, the energy consumption is reformulated including a penalty $\psi$ on the electrical energy consumption. The objective function formulation then looks as:

$$
\min _{(x, u)} \int_{t_{0}}^{t_{f}, f i x} \operatorname{Jerk}+\delta \times\left(E_{m_{f}}+\psi \times E_{\text {gen }}\right) d t
$$

The problem in (19) is solved with various combinations of $\delta$ and $\psi$ penalties.

Finally, the different OCP formulations with the objective functions mentioned above become:

$$
\begin{aligned}
& \text { Objective function in (15) or (16) or (17) or (19) } \\
& \text { subject to: } \quad \dot{x}=f(x, u) \\
& \text { Constraints in (13) and (14) }
\end{aligned}
$$

\subsection{Numerical solution of optimal control problems}

To solve the formulated OCP in the previous section, a direct multiple shooting method using CasADi software package (Andersson, 2013) is used. The dynamics in each discretization interval are forward integrated using a 4 step Runge-Kutta integrator. After discretization of constraints, objective function and the dynamics, a nonlinear programming problem (NLP) is formulated and solved using IPOPT (Wächter and Biegler, 2006) to obtain the optimal controls and corresponding state transients.

To ensure that the solutions are not affected by the number of discretization intervals, the problem is solved with different values. It is seen that the transients remain unchanged for intervals close to and more than 300 so this is chosen as the number of discretization intervals.

\section{Optimal control results}

\subsection{Extreme transients}

To show the importance of finding a compromise between time, Jerk, and energy objectives, the state and control transients are first presented for extreme cases where only one of these are considered in the optimization. These transients are obtained by solving for (15), and (16) and (17) the latter two with final time locked $t_{f, f i x}=2 \mathrm{~s}$.

The min $\mathrm{T}$ transients, illustrated in Figure 1, are very oscillatory at the beginning for all controls and such control strategy would have severe negative impacts on the passenger comfort as well as the life length of genset and driveline components. For the bus to be able to smoothly continue its movement after reaching the final speed, the twist angle in the driveshaft should match the required torque and acceleration at wheels. In min $\mathrm{T}$ transients, due to the high deflection in the driveshaft, the vehicle speed even at the end of the acceleration is still increasing at a 
high rate. A transition from this high acceleration to a low acceleration would be undesirable in terms of passenger comfort standards.

The minimum energy transients are less oscillatory but diesel engine power is not used for vehicle propulsion and all required power is provided only by the electric motor. The very low engine speed at the end of the minimum energy transients, increases the risk of engine stall when the diesel engine is going to take over the power production after the acceleration which makes the controls less applicable in real world applications.

For min Jerk transients, the undesirable non-smooth speed transition at the end time is similar to the min $\mathrm{T}$ case. The controls are less oscillatory compared to the min $\mathrm{T}$ case, but the bang-bang looking controls are what the manufacturers are less willing to implement because of the issues with component wear and durability accompanied with such control strategies.

Considering the mentioned drawbacks, these solutions are considered extreme and less applicable for control design in real world applications. In the following sections, the transients obtained by the suggested methodology for finding proper compromise between time-energy-Jerk objectives are presented and analyzed.

\subsection{Compromise between time, Jerk and en- ergy}

Figure 2 shows the trade-off between time and Jerk objectives calculated by solving (16) as stated in Section 3.2 for three different road slopes. The Jerk in min $\mathrm{T}$ solution, calculated by solving (15), is extensively larger and therefore it is not included in the trade-offs. However, the Jerks in Figure 2 are normalized with respect to the largest Jerk belonging to the min $\mathrm{T}$ solution of the 0 degree slope case which is referred to as Jerk $k_{\max }$. It is seen that the Jerk can be significantly decreased compared to the min $\mathrm{T}$ solution for all road slopes. It is desirable to have small Jerk during operation, specifically in a city bus application. Therefore, a duration of 2 [s] where the Jerk approach near zero values for all road slopes is chosen as the fixed time duration for which the energy-Jerk trade-off is calculated.

\subsection{Jerk-Energy trade-off}

To investigate the energy balance during the genset operation, different energy components and fuel conversion efficiency are defined as follows:

$$
\begin{aligned}
E_{\text {tot }} & =E_{m f}+E_{g e n} \\
E_{\text {loss }} & =E_{m f}+E_{e} \\
E_{e} & =\int_{t_{0}}^{t_{f}} M_{e} \omega_{e} d t \\
\eta_{m f, c o n v} & =\frac{E_{e}}{E_{m f}}
\end{aligned}
$$

where $E_{e}$ represents the net energy from the diesel engine which is used for acceleration, and $E_{\text {loss }}$ represents the losses such as engine friction and pumping work.
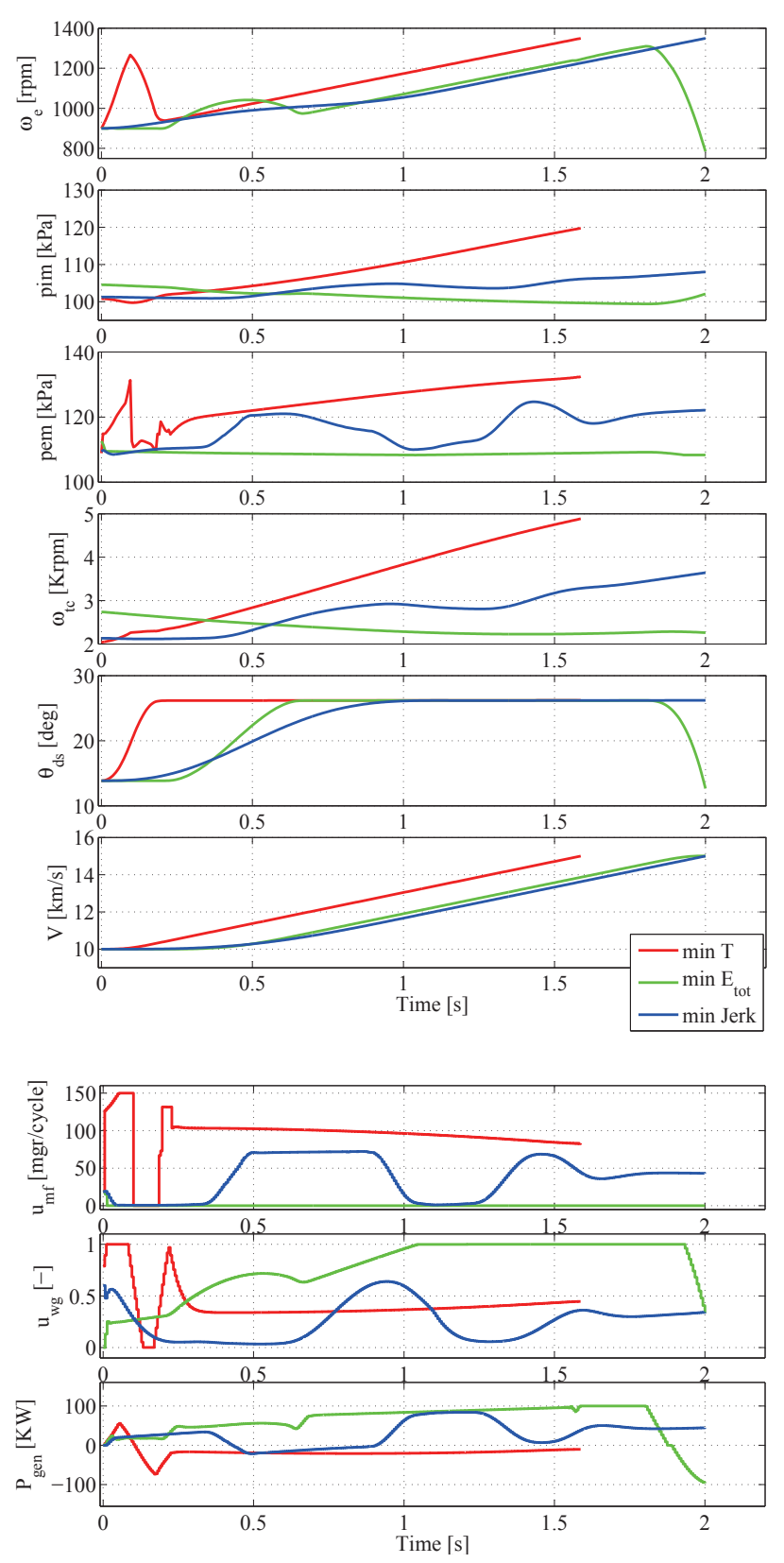

Figure 1. Optimal state and control transients for the extreme cases during acceleration $\left(E_{t o t}=E_{m f}+E_{g e n}\right)$.

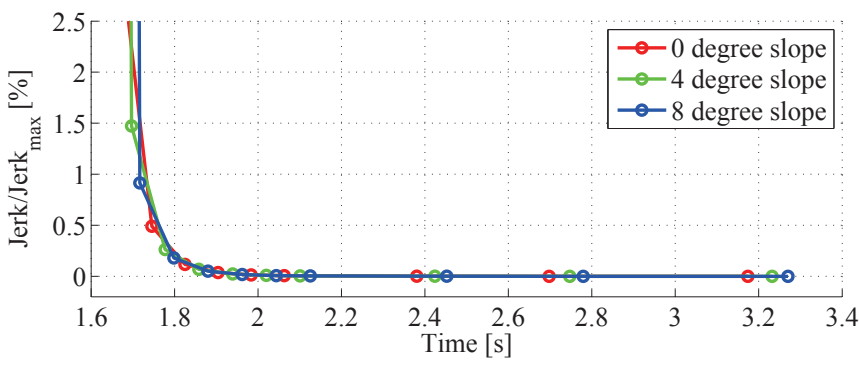

Figure 2. Trade-off between Jerk and time objectives.

To obtain the trade-off between Jerk and energy for different road slopes, (19) is solved while increasing $\delta$ and 
$\psi$. The results for the three road slopes are presented in Figure 3. Reminding that the purpose of applying energy penalties is to avoid "extreme" low Jerk transients, a point with slightly increased Jerk on the trade-offs is chosen as a "candidate" case for which the energy balance is presented in Figure 4. Independent of what power is required for acceleration, the total required energy shows a decreasing trend when the penalty $\delta$ is increased. Also, when the penalty $\psi$ is increased, meaning that the usage of electrical energy becomes more costly in the (19), more power is delivered by the diesel engine which has a low efficiency and therefore the total required energy for vehicle acceleration increases. As seen in Figure 4, for larger $\psi$ values, less electrical energy is used and when total required energy for acceleration is low, the 0 degree case, diesel engine power is even used to produce electrical energy in addition to vehicle acceleration.

In case of the 0 degree slope, the required energy for the $\psi=0$ remains unchanged for all $\delta$ values. This is because this operating condition requires smaller amount of energy compared to other cases while $\psi=0$ in (19) implies that it does not have any cost to use electrical energy. Low efficiency of the diesel engine compared to the generator and the cheapness of electrical energy makes it optimal to perform the acceleration using only the electrical energy with no regard to the penalty $\delta$ on total energy consumption. This can be verified comparing the $\psi=0$ energy balance for the three road slopes in Figure 4.

The first time diesel power is used for acceleration in the 0 degree slope is at $\psi \approx 2.05$. At this operating condition, a sudden decrease in $E_{t o t}$ takes place according to Figure 4. Moving from the first point on the $\psi=2.05$ to the second point of the curve in Figure 3, the increase in $\delta$ makes the contribution from the energy term larger than the Jerk term in (19). As a result, a higher efficiency in energy consumption is favored. Since usage of the fuel energy accompanies high losses, achieving higher total efficiency is facilitated by altering the contribution of energy sources from very low electrical energy usage, similar to $\psi=2.08$ in the 0 degree slope of Figure 4, into nearly equal contribution from the electrical and fuel energy sources, in $\psi=2.05$.

Considering the fuel conversion efficiencies presented in Table 3, the efficiency is lower at low loads corresponding to the 0 degree slope and when electrical energy is cheaper to use (smaller $\psi$ values). Other than this, an efficiency close to $40 \%$ is maintained at different loading conditions.

Table 3. Fuel conversion efficiency.

\begin{tabular}{cc|ccccc}
\hline road slope & $\psi$ & 0 & 2.05 & 2.08 & 2.5 & 4 \\
0 [deg] & $\eta_{m f, \text { conv }}[\%]$ & -16.6 & 34.7 & 39.9 & 40.6 & 40.3 \\
\hline \hline road slope & $\psi$ & 0 & 2.02 & 2.05 & 2.2 & 4 \\
4 [deg] & $\eta_{m f, \text { conv }}[\%]$ & 28.74 & 38.02 & 40.16 & 40.86 & 40.13 \\
\hline \hline road slope & $\psi$ & 0 & 2.1 & 2.2 & 2.5 & 4 \\
8 [deg] & $\eta_{m f, \text { conv }}[\%]$ & 39.6 & 40.94 & 41.08 & 40.59 & 39.43 \\
\hline
\end{tabular}

\subsection{Efficient state and control transients}

The efficient state and control transients for the candidate points of the 0 degree slope case in Figure 3 are presented in Figure 5. 

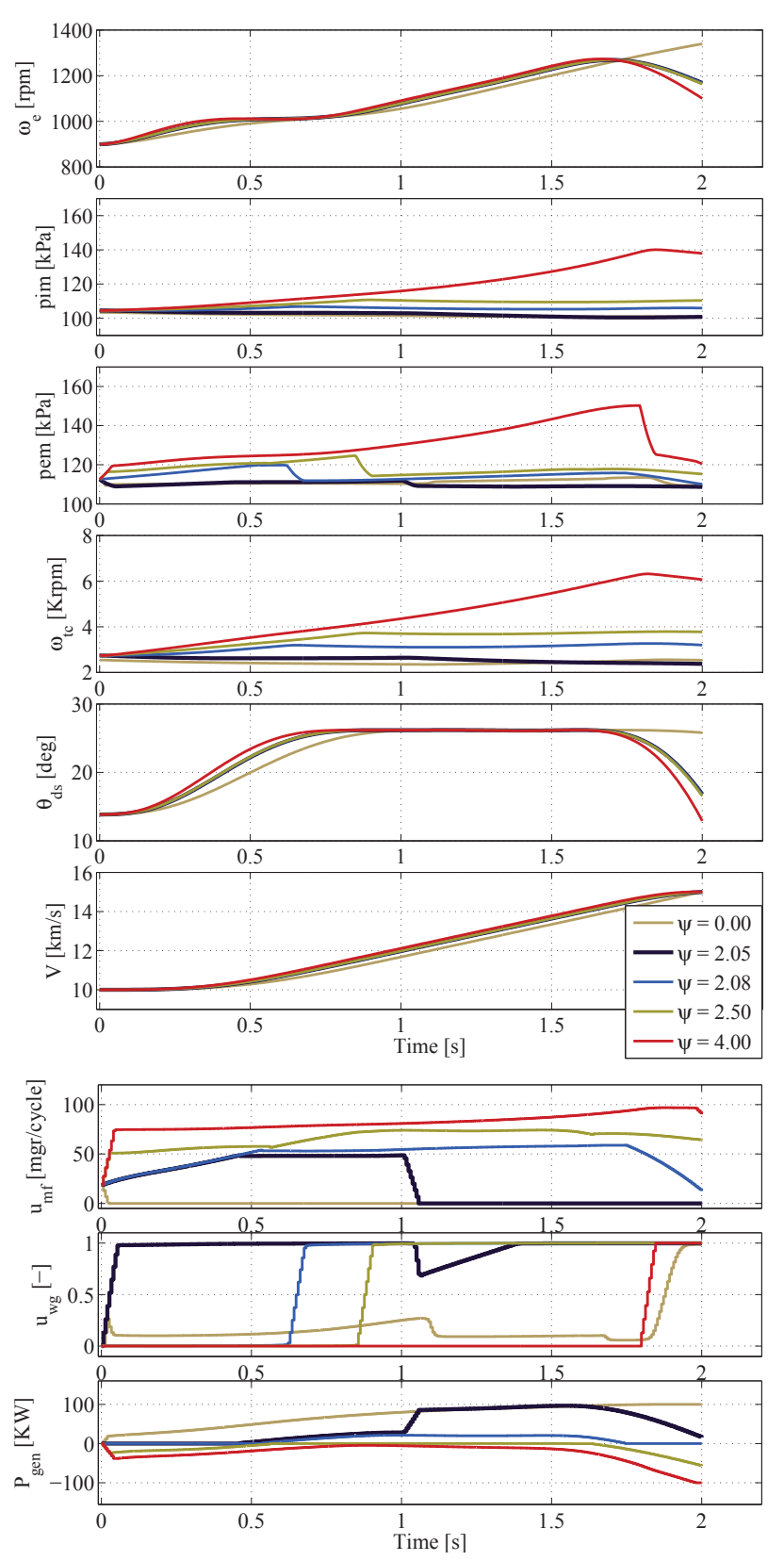

Figure 5. Optimal state and control transients during tip-in calculated for the candidate points in Figure 3.

According to the figures, as the cost for using electrical energy increases (larger $\psi$ ), the diesel engine transients are largely changed. for example comparing the $\psi=0$ and $\psi=4$ cases, when $\psi=0$ fuel is cut-off, diesel engine power is not used and only electrical power accelerates the vehicle. But for $\psi=4$, not only usage of the costly electrical energy is avoided but also parts of diesel engine power is used to store electrical energy at the end of the acceleration. Increasing the fuel conversion efficiency in the diesel engine operation is the main priority here and for that, fuel injection is selected such that the engine operates at the smoke limit delivering as large power as possible. This is similar to the discussion in (Sivertsson and Eriks- son, 2015a) and (Sivertsson and Eriksson, 2015b) stating that the smoke limit dictates the solution during large parts of the transients. After an initial high power production which has facilitated fast vehicle acceleration, the wastegate which has been kept closed until this point, is opened at ca 1.7 [s] to lower the pumping work losses. Vehicle acceleration is reduced and less power from the engine is required to meet the final speed constraint. Instead, the engine power is used to build up electrical energy.

Considering the points mentioned about the extreme transients such as oscillatory controls or large acceleration at the end time, according to Figure 5, the transients for the $\psi \approx 2.05$ can be an example of improved control strategy with simple control transients and smooth vehicle speed transients at end time.

\section{Conclusions}

Optimal control of a diesel-electric powertrain during a tip-in acceleration is analyzed while importance of proper objective function formulation is highlighted. The extreme transients resulting from minimization of only jerk or time or energy are presented and the drawbacks in terms of oscillatory control signals are discussed. It is shown that by calculation of the trade-off between time and Jerk, low Jerk transients can be obtained. By applying penalties on energy consumptions in the Jerk minimization problem and solving for various fuel and electric energy weights in the objective function formulation, energy efficient transients are obtained. The calculated transients using this approach are presented which are simpler and more insightful for control design in real world applications. At the same time, the proposed controls maintain low Jerk and energy consumption compared to the extreme cases.

\section{Acknowledgment}

The support and feedback from engineers at SCANIA CV $\mathrm{AB}$, and funding from Swedish Energy Agency is gratefully acknowledged.

\section{References}

Joel Andersson. A General-Purpose Software Framework for Dynamic Optimization. $\mathrm{PhD}$ thesis, Arenberg Doctoral School, KU Leuven, Department of Electrical Engineering (ESAT/SCD) and Optimization in Engineering Center, Kasteelpark Arenberg 10, 3001-Heverlee, Belgium, October 2013.

A Haj-Fraj and F Pfeiffer. Optimal control of gear shift operations in automatic transmissions. Journal of the Franklin Institute, 338(2):371-390, 2001.

A Haj-Fraj and F Pfeiffer. A model based approach for the optimisation of gearshifting in automatic transmissions. International journal of vehicle design, 28(1):171-188, 2002.

V. Nezhadali and L. Eriksson. Optimal control of engine controlled gearshift for a diesel-electric powertrain with backlash. In AAC'16 - 8th IFAC Symposium on Advances in Automotive Control, Kolmården, Sweden, 2016. 
Magnus Pettersson and Lars Nielsen. Gear shifting by engine control. IEEE Transactions Control Systems Technology, 8 (3):495-507, May 2000.

Martin Sivertsson and Lars Eriksson. Time and fuel optimal power response of a diesel-electric powertrain. In $E$ COSM'12 - IFAC Workshop on Engine and Powertrain Control, Simulation and Modeling, Paris, France, October 2012a.

Martin Sivertsson and Lars Eriksson. Optimal step responses in diesel-electric systems. In Mechatronics'12 - The 13th Mechatronics Forum International Conference, Linz, Austria, September 2012b.

Martin Sivertsson and Lars Eriksson. Modeling for optimal control: A validated diesel-electric powertrain model. In SIMS 2014 - 55th International Conference on Simulation and Modelling, Aalborg, Denmark, 2014.

Martin Sivertsson and Lars Eriksson. Optimal transient control trajectories in diesel-electric systems-part 1: Modeling, problem formulation and engine properties. Journal of Engineering for Gas Turbines and Power, 137(2), February 2015a.

Martin Sivertsson and Lars Eriksson. Optimal transient control trajectories in diesel-electric systems-part 2: Generator and energy storage effects. Journal of Engineering for Gas Turbines and Power, 137(2), February 2015b.

Standardised On-Road Test cycles (SORT). http: //www.uitp.org/sites/default/files/ documents/Knowledge/UITP_Project_SORT_ GAS_20151021.pdf, Last accessed April 2016.

Andreas Wächter and Lorenz T Biegler. On the implementation of an interior-point filter line-search algorithm for largescale nonlinear programming. Mathematical programming, 106(1):25-57, 2006. 